\title{
Transcriptional Functions of DNA Repair Proteins Involved in Premature Aging
}

\author{
Assfalg, Robin and Iben, Sebastian \\ Department of Dermatology and Allergic Diseases \\ University of Ulm \\ Germany
}

\section{Introduction}

Premature aging diseases or progerias are rare genetical disorders displaying symptoms of the aging body early in life or even in childhood. They are called segmental because they show some, but not all features of aging. There is the progeria of the adult, Werner syndrome and, more severe because limiting lifespan to the first or second decade, the progerias of the childhood, Cockayne syndrome, trichothiodystrophy and HutchinsonGilford syndrome. With the exception of Hutchinson-Gilford syndrome, the progerias are caused by recessive mutations. Cockayne syndrome and trichothiodystrophy are polygenic disorders- the recessive mutation in five respective three different genes can cause the same devastating phenotype. All genes of a polygenic disorder may function in a critical redundant pathway. The identification of these pathways is topic of intensive labour in different laboratories for more than one reason. First, the identification of the molecular defects will help us to treat these diseases. Second, as these diseases mimic the normal aging process, understanding these diseases will strengthen our understanding of aging in general. Third, as these disorders display accelerated aging, the underlying pathways may be critical for the rate of aging and may help us to slow aging respectively allow us to affect healthy aging. Aging is believed to be due to the accumulation of molecular and macromolecular damage (Kirkwood, 2010), thus accelerated aging might be caused by a higher damage rate or by an impairment of counteracting pathways as repair mechanisms. The later assumption is generally believed to be the explanation for the accelerated aging seen in progerias, defects in macromolecular repair pathways, especially in DNA-repair pathways are generally considered as being causal for accelerated aging. Although there is ample evidence that aging is accompanied by macromolecular damage and DNA damage in particular, the causal connection between DNA damage and tissue or organismal aging is far from understood. Here the investigation of progerias is able to fuel our understanding of the mechanisms of aging as most of the involved genes play roles in different DNA repair pathways. But it is not so simple because all the progeria genes display multiple functions in the cells and are also involved in the regulation of gene expression by acting as basal transcription factors or as chromatin modifying enzymes. The discovery of the transcriptional function of DNA repair factors was accompanied by the hypothesis that accelerated aging could also be caused by alterations in gene expression mechanisms (Drapkin et al, 1994, Guzder et al, 1994). Since then the "transcriptional" versus the "DNA 
repair" hypothesis were intensively discussed and today there is a lot of evidence for the involvement of both pathways in the pathogenesis of premature aging (Chalut et al,1994, Winkler and Hoeijmakers,1998, Bergmann and Egly 2001, deBoer et al 2002). Transcription of DNA by the RNA polymerases serves as a DNA damage sensor and recruits DNA repair proteins to sites of DNA damage. Moreover, hitherto as pure DNA repair factors recognized proteins turned out to be involved in chromatin remodeling and epigenetic modulation of gene expression of undamaged DNA (Schmitz et al, 2009, LeMay et al, 2010). Thus repair of DNA and gene expression at the level of transcription are intimately structurally and functionally linked. Here we review the current knowledge about transcriptional functions of DNA repair proteins involved in the pathogenesis of progerias.

\section{Premature aging syndromes}

\subsection{Werner syndrome}

Werner syndrome (WS) or the progeria of the adult, is an autosomal recessive genetic instability and cancer predisposition syndrome that mimics premature aging. WS patients lack the pubertal growth spurt and develop bilateral cataracts, premature graying and loss of hair and scleroderma-like skin changes already beginning in the second decade of life. Patients have an elevated risk of age-associated diseases as atherosclerotic cardiovascular disease, diabetes mellitus, osteoporosis and cancer. Life expectancy is shortened to 47 years.

The WS gene WRN encodes a member of the RecQ helicase protein family and posseses an additional 3'-5'exonuclease domain. WRN is involved in different DNA metabolizing pathways as DNA repair and replication, telomere maintenance and transcription (Rossi et al, 2010, Chu and Hickson, 2009, Ding and Shen 2008). WRN deficient cells display a telomere lagging strand replication defect and karyotypic instability associated with short telomers (Crabbe et al, 2004, Crabbe et al, 2007). Moreover, premature aging in mice deficient for WRN is dependent on telomere shortening in the telomerase RNA subunit TERC deficient background (Chang et al, 2004). These double knockout mice also show a hallmark of cells derived from Werner syndrome patients- premature senescence. Whereas fibroblasts from normal donors enter the stage of irreversible division stop, replicative senescence, after 60 population doublings, cells from Werner syndrome patients enter senescence after 15-20 population doublings (Faragher et al, 1993). Thus it seems that the role of the WRN protein at the telomeres might be critical to protect us from premature aging. Nonetheless, there are host of questions arising. It is not clear if replicative senescence is responsive for aging pathologies, although markers of replicative senescence accumulate in aged skin of baboons (Herbig et al 2006). Studies on telomere length dynamics in Werner syndrome cells revealed that telomeres did not erode faster than in normal cells (Baird et al, 2004). Expression of telomerase in Werner syndrome cells can overcome premature senescence (Wyllie et al, 2000, Choi et al, 2001), but inhibition of the stress-activated kinase p38 by the compound PD203580 also extended the replicative lifespan of WS cells to that observed in normal fibroblasts (Davis et al 2005). These observations substanstiate that our knowledge about the pathophysiology of Werner syndrome is still limited and that alternative concepts also deserve attention.

The first indication of the involvement of RecQ helicases in gene expression came from studies of the yeast WRN homolog sgs1. As in WS, the sgs1 deletion decreases the average life span of cells and accelerates aging (Sinclair et al, 1997). Conditional mutation of sgs1 in a yeast strain lacking the helicase srs 2 is followed by a drastical inhibition of DNA replication 
and RNA polymerase I transcription. The authors concluded that the replication defect could contribute to the genomic instability of WS, whereas impaired ribosomal RNA chain elongation may render RNA polymerase I prone to pausing that could trigger the formation of double strand breaks. Repair by nonhomologous end-joining could result in the accumulation of deletions in the genomic rDNA and contribute to premature aging in WS (Lee et al, 1999). Shiratori and co-workers showed that in human WS cells RNA polymerase I transcription is reduced and can be restored by wildtype WRN. Moreover, nucleolar localization of WRN is dependent on ongoing RNA polymerase I transcription. WRN can be co-immunoprecipitated with RNA polymerase I. In humans, the decreased transcriptional rate of rRNA could be the primary molecular defect causing the premature aging phenotype in WS patients, the authors speculated (Shiratori et al, 2002). Another study with human WS cells described, that the stimulation of RNA polymerase I transcription by some growth factors is impaired in WS cells. Moreover, WRN acts as a transcription factor and stimulates the step of promoter clearance of RNA polymerase I transcription. Chromatinimmunoprecipitations revealed that WRN binds to quiescent and unmethylated rDNA, implicating a role of WRN in epigenetic regulation of RNA polymerase I transcription. Taken together, this study implicated that WRN acts as a growth factor dependent transcription factor of RNA polymerase I and may prevent inactivation of rDNA genes in the absence of growth factors (Lutomska et al, 2008). These mechanisms may contribute to the lack of the pubertal growth spurt, impaired wound healing and premature aging in WS patients.

Beside RNA polymerase I transcription several publications show that WRN influences gene expression by RNA polymerase II. One study from 1999 describes a significant reduction of RNA polymerase II transcription in WS cells. This is reflected in in vitro transcription and can be rescued by addition of wildtype but not mutant WRN. Moreover a 27aa repeated sequence in the WRN gene was identied as a strong transcriptional activator domain. The transcription defect in WS cells may be global or may affect certain genes or categories of genes within the genome (Balajee et al, 1999). The later hypothesis is supported by a report showing that the transcriptional activator function of p53 is stimulated by WRN (Blander et al, 1999). Gene expression profiling comparing cells from young donors and cells from old donors with WS cells unravelled that mutation of WRN affects the expression of certain genes within the genome. Moreover transcription alterations in WS were strikingly similar to those in normal aging (Kyng et al, 2003). These findings validated WS as a model disease for aging research and established, that WRN influences the expression of certain genes within the genome. To investigate if the observed changes in the transcriptome are due to the direct loss of WRN or are secondary consequences of genomic instability, Turaga et al used short-term siRNA based knockdown of WRN. This was sufficient to trigger an expression profile resembling fibroblasts established from old donor patients and identified genes involved in 14 distinct biological pathways to be affected by loss of WRN. It is conceivable that WRN might associate with chromatin and affect the activity of classical transcription factors (Turaga et al, 2009). A recent report also used microarray expression analysis to investigate if RecQ helicases and WRN in particular regulate genes enriched in G-quadruplex DNA, a family of non-canonical nucleic acids structures formed by certain Grich sequences. RecQ helicases can unwind these structures in vitro and in vivo. In fact the authors found significant associations between loci that are regulated in WS and loci containing potential G-quadruplex forming sequences. These findings indicate that WRN can regulate transcription globally by targeting G-quadruplex DNA (Johnson et al, 2010). 
Taken together there is ample evidence that the WRN protein influences transcription by RNA polymerase I and II and is involved in gene regulation of certain genes that are also regulated throughout the normal aging process and in the globally regulation of genes with G-quadruplex forming sequences.

\subsection{Cockayne syndrome}

Cockayne syndrome is an autosomal recessive neurodegenerative disorder characterized by progressive growth failure, retinal degeneration, cataracts, sensorineural deafness, mental retardation, and photosensitivity (Nance and Berry, 1992, Laugel et al, 2009). Cataracts, loss of retinal cells, neurological degeneration and cachexia are prominent premature aging symptoms of this disorder followed by infant death. Cockayne syndrome is caused by mutations in CSA and CSB genes and rare combinations with the cancer prone skin disease xeroderma pigmentosum and complementation groups $X P B, X P D$ and $X P G$ have been described. All five genes are involved in repair of helix-distorting lesions of DNA by the nucleotide excision repair pathway (NER). Damage recognition mechanisms differ in the subpathways transcription-coupled repair (TCR) in which CSA and CSB are involved and global genomic repair (GGR). Both pathways of damage recognition flow into a common DNA repair mechanism. Premature aging in Cockayne syndrome is commonly attributed to defective transcription coupled repair (Hoeijmakers, 2009), although this view raises a plethora of questions. There are multiple mutations in NER proteins described (XPA, XPB, $\mathrm{XPD}, \mathrm{XPF}, \mathrm{XPG}$ ) that completely impair both branches of this DNA repair pathway, but are not followed by premature aging but cause the severe cancer prone skin disease xeroderma pigmentosum. Xeroderma pigmentosum patients, when shed from UV-light do not develop the premature aging traits of Cockayne syndrome although the same type of DNA lesions remain unrepaired and should accumulate and disturb cellular fidelity. Thus transcription coupled repair might also be responsive for the repair of hitherto undefined DNA lesions that compromise transcription (Laugel et al, 2009). It is conceivable that all five genes that, when mutated cause Cockayne syndrome, are involved in a critical redundant function that protects us from accelerated aging. This function is not identified yet. Transcription-coupled repair is responsible for the recruitment of the repair machinery to the transcribed strand of active genes. It has been shown, that nearly all factors involved in transcription coupled repair are coincidental participating in basal transcription mechanisms thus raising the possibility that premature aging might be due to aberrant transcription. This hypothesis is discussed since two decades without a definitive answer. Here, we would like to review the current knowledge about the transcriptional functions of the five proteins involved in the pathogenesis of Cockayne syndrome.

\subsubsection{Cockayne syndrome protein A (CSA)}

As the CSA gene was identified, the interaction of the corresponding protein with CSB and the TFIIH subunit p44 were described. TFIIH, beside being essential for Nucleotide Excision Repair (NER) of UV damaged DNA, is a general transcription factor of RNA polymerase II (see below). Thus the authors proposed a transcriptional function of CSA (Henning et al, 1995). In vitro transcription studies with a template bearing oxidative lesions showed a reduced RNA polymerase II transcription in nuclear extracts of CSA cells, that could be rescued by the overexpression of CSA indicating a transcriptional function of CSA (Dianov et al, 1997). However, microinjection of CSA antibodies in cells reduced the repair capacity 
of the cells, but did not influence the rate of transcription by RNA polymerase II (van Gool et al, 1997). Since then additional evidence for a direct involvement of CSA in transcription was not found.

\subsubsection{Cockayne syndrome protein B (CSB)}

Mutations in the ERCC6/CSB gene are responsible for $62 \%$ of Cockayne syndrome cases (Laugel et al, 2009). The ERCC6 gene product belongs to the family of SWI/SNF chromatin remodeling enzymes (Troelstra et al, 1992). ATP-dependent chromatin remodeling enzymes coordinate changes in chromatin structure to help regulate transcription. This structure of CSB implies a regulatory role in transcription. The first study investigating a transcriptional role of CSB used in vivo labeling and permeabilisation of cells to show that CSB mutant cells exhibit a severely reduced RNA polymerase II transcription that could be restored by addition of CSB. As transcription of chromatin from permeabilized cells represent the elongation activity of RNA polymerase II, the authors proposed a role of CSB in transcription elongation (Balajee et al, 1997). Using microinjection of antibodies against CSB, van Gool et al did not detect an inhibition of transcription by all three RNA polymerases measured by labelled thymidine incorporation However, CSB cofractionates with RNA polymerase II over chromatographic columns and RNA polymerase II can be coimmunoprecipitated with CSB (van Gool et al, 1997). As these complexes do not contain initiation factors of RNA polymerase II, the authors speculated that CSB might be involved in a non-essential step of transcription elongation. Another study confirmed the interaction of RNA polymerase II with CSB and could convincingly show, that CSB stimulates elongation by RNA polymerase II in vitro (Selby and Sancar, 1997). If this function of CSB is relevant for transcription elongation in vivo, the study by van Gool et al should have detected a reduction of transcription by microinjection of CSB antibodies. The recruitment of CSB to RNA polymerase II elongation complexes in vitro was also demonstrated by other investigators (Tantin et al, 1997) but the in vivo relevance of this interaction was not studied. CSB mutant cells were found to exhibit metaphase fragility of highly transcribed genes of RNA polymerase II and III that are coding for structural RNAs. The authors proposed that CSB might play a role in transcription elongation of these genes and lack of CSB would be followed by stalled polymerases inducing metaphase fragility ( $\mathrm{Yu}$ et al, 2000). ATPdependent chromatin remodeling activity of CSB was substantiated in another study, thus implicating that CSB may play a role in facilitating transcription by RNA polymerase II through pause sites on natural chromatin templates in vivo by modulating nucleosome structure on DNA. It is possible that defective chromatin rearrangements during DNA repair or transcription may contribute to the severe clinical symptoms of CS patients (Citterio et al, 2000). A study performed in Saccharomyces cerevisiae provided in vivo evidence for a role of rad26, the counterpart of the CSB gene, in transcription elongation by RNA polymerase II. Under conditions requiring rapid synthesis of new mRNAs, growth is considerably reduced in cells lacking rad26. These findings implicate a role for CSB in transcription elongation, and they strongly suggest that impaired transcription elongation is the underlying cause of the developmental problems in CS patients (Lee et al, 2001).

The same authors showed that the CSB homolog rad26 plays a role in promoting transcription by RNA polymerase II through bases damaged by the alkylating agent MMS. Transcription through these bases is severely inhibited in rad26 cells lacking both the NER (nucleotide excision repair) and BER (base excision repair) pathways required for the removal of these lesions (Lee et al, 2002). This report demonstrates a transcriptional function 
of CSB independent from DNA repair and, moreover, fit to the observation, that nuclear extracts from CS cells of three complementation groups exhibit reduced RNA polymerase II in vitro transcription only on oxidised template (Dianov et al, 1997).

Microarray analysis of gene expression profiles did not identify significant differences in gene expression between CSB deficient and transfected cells indicating that CSB does not function as a gene specific transcription factor (Selzer et al, 2002). Bradsher et al reported a novel aspect of CSB as a component of RNA polymerase I transcription in the nucleolus. CSB was localized to nucleoli and isolated in a complex with RNA polymerase I, transcription initation factors of RNA polymerase I, TFIIH and XPG. CS mutations in TFIIH subunits rendered this complex instable and stability of this transcription competent complex was speculated to contribute to Cockayne syndrome phenotype. RNA polymerase I transcription was reduced in CSB mutant cells and restored by transfection of CSB (Bradsher et al, 2002). This report is inasmuch interesting as it describes a functional complex of 4 from 5 proteins that, when mutated cause Cockayne syndrome, indicating that rDNA transcription by RNA polymerase I might be the redundant function of the CS proteins whose failure causes premature aging.

Microarray analysis after oxidative stress revealed that there is a bundle of genes that is differently regulated after $\mathrm{H}_{2} \mathrm{O}_{2}$ treatment of CSB deficient and CSB competent cells. If the identified genes are directly regulated by CSB was not further specified (Kyng et al, 2003).

Confocal microscopy and quantitative digital image analysis of different photobleaching (FRAP) procedures showed transient interactions of CSB with the transcription machinery, which are prolonged when RNA polymerases are arrested at sites of DNA damage. Active RNA polymerase II could be immunopurified with CSB, but no transcription factors were found to be associated (van dem Boom et al, 2003). The CSB function in transcription was to this timepoint always linked to transcription elongation. A novel study discovered functions of CSB upstream of transcription initiation. The authors unveiled the crucial role played by CSB in the transcription initiation of a certain set of protein coding genes after UV irradiation. CSB cells cannot transcribe even nondamaged genes if the cells were previously UV irradiated. The recruitment of TBP, which is supposed to initiate transcription, was severely decreased; also, the recruitment of TFIIB was almost absent. Furthermore, histone $\mathrm{H} 4$ acetylation does not occur properly, highlighting a defect in one of the earlier events of the transcriptional process. The fact that CSB associates mainly with the unphosphorylated RNA pol IIA and the serine 5 phosphorylated RNA pol IIO, strongly supports a role for CSB during the first phases of the transcription reaction (Proietti-Di-Santis et al, 2006). Although earlier microarray analysis did not yield gene expression differences in CS-cells (Selzer et al,2002), refined methodology using microarrays in combination with a unique method for comparative expression analysis found many genes regulated by CSB. Remarkably, many of the genes regulated by CSB are also affected by inhibitors of histone deacetylase and DNA methylation, as well as by defects in poly(ADP-ribose)-polymerase function and RNA polymerase II elongation. This data indicate a general role for CSB protein in maintenance and remodeling of chromatin structure and suggest that CS is a disease of transcriptional deregulation caused by misexpression of growth-suppressive, inflammatory, and proapoptotic pathways (Newman et al, 2006). In vitro transcription analysis using a reconstituted transcription system showed, that bypass of different oxidative lesions in the template requires elongation factors like CSB, thus again evaluating the initial observation by Dianov et al, that CSB deficient cells exhibit reduced RNA polymerase II transcription on oxidised template (Charlet-Berguerant et al, 2006). 
Extending the findings of Newman et al, that CSB influences chromatin remodeling to transcription of RNA polymerase I, Yuan et al demonstrated in an intricate analysis that transcription activation of RNA polymerase I is dependent on CSB. CSB is recruited to active rDNA repeats by TTF-I bound to the promoter-proximal terminator $\mathrm{T}_{0}$. Depletion of CSB by siRNA impairs the formation Pol I preinitiation complexes and inhibits rDNA transcription. CSB recruits G9a that methylates histone $\mathrm{H} 3$ on lysine 9 (H3K9) in the prerRNA coding region. The results demonstrate that the functional cooperation between CSB and G9a is important for efficient pre-rRNA synthesis (Yuan et al, 2007). This study integrates findings of several above mentioned publications. A gene specific regulatory function upstream of transcription initiation was mechanistically deciphered and a chromatin organisation mode of CSB was described in detail.

Analysis of in vitro transcription by RNA polymerase I revealed that CSB plays a role as an elongation factor in rDNA transcription and that truncated CSB still localizes to the rDNA repeats in vivo. Truncated CSB actively represses in vitro transcription of RNA polymerase I thus providing an explanation for the observation that a null mutation in CSB is not necessarily followed by CS (Horibata et al, 2004) whereas truncating mutations are devastating (Lebedev et al, 2008).

CSB is also a critical mediator of the hypoxic response and influences binding of the general transcription factors and RNA polymerase II in a gene-specific manner in response to hypoxia as demonstrated by chromatin-immunoprecipitation analysis. CSB binds to p53 and might also influence its transcriptional activity, the authors speculated (Filippi et al, 2008). Thus it becomes evident that CSB is not only an elongation factor of RNA polymerase II but exhibits gene regulatory functions in a gene-specific manner.

The reviewed studies clearly show that CSB is an elongation factor of transcription by RNA polymerase I and II and that CSB facilitates transcription through damaged bases. Additional, CSB functions upstream of initiation by RNA polymerase I and II by recruiting chromatin modifying cofactors or by remodeling chromatin itself in a gene specific manner.

\subsubsection{TFIIH}

TFIIH is a multisubunit complex composed of ten subunits. It habors three enzymatic activities, two ATP dependent helicases of opposite orientation, XPB and XPD and the cyclin dependent kinase cdk7. Mutations in the XPB and XPD helicases are followed by the skin cancer prone xeroderma pigmentosum syndrome but also by the premature aging syndromes Cockayne and trichothiodystrophy. Mutations in the recently discovered tenth subunit p8/TTDA destabilize TFIIH and is followed by trichothiodystrophy. Xeroderma pigmentosum is characterized by a 1000 fold elevated skin cancer risk, the german term "Mondscheinkinder" translated "moonshine-children" denominates the fact that the failure of Nucleotide excision repair (NER) renders the skin of affected children so sensitive to UVinduced DNA damage and consecutive development of skin destructive cancers that they need to be shed totally from UV-light by special clothing. Then they develop normally. The ATPase of XPB and XPD helicase activity of TFIIH are necessary for the unwinding of the damaged DNA strand that can then be cleaved and resynthesized. Thus highly mutagenic DNA lesions persist in the genome when the helicase functions of TFIIH are reduced or inactivated by mutations. The second main function of TFIIH is as a general transcription factor of RNA polymerase II. General or basal transcription factors are needed at every protein coding gene for bending of the promoter, positioning of the polymerase or promoter opening as through the ATPase activity of the XPB subunit of TFIIH (Kim et al, 2000, 
Douziech et al, 2000). TFIIH has also been reported to play a postinitiation role in transcription by RNA polymerase I, the key step of ribosomal biogenesis, that accounts for up to $60 \%$ of ongoing transcription in a growing cell (Iben et al, 2002).

Xeroderma pigmentosum is due to unrepaired DNA damage, whereas premature aging in Cockayne syndrome and trichothiodystrophy might be caused by transcriptional deficiencies. Several studies addressed this hypothesis and an unequivocal answer to the question "is it repair or transcription?" has not been delivered yet.

Trichothiodystrophy mice with a mutation in XPD reflect to a remarkable extent the human disorder, including brittle hair, developmental abnormalities, reduced life span, UV sensitivity, and skin abnormalities. The cutaneous symptoms are associated with reduced transcription of a skin-specific gene strongly supporting the concept of TTD as a human disease due to inborn defects in basal transcription. To explain the characteristic hair and skin abnormalities of TTD, TTD-type XPD mutations may alter the XPD conformation and in this way affect the stability of the TFIIH complex. Under normal conditions, de novo synthesis of TFIIH is thought to compensate for the reduced half-life. However, in terminal differentiating tissues where de novo synthesis gradually declines, the mutated TFIIH might get exhausted before the transcriptional program has been completed (de Boer et al, 1998). These authors describe that late in the differentiation pathway and thus gene-specific transcription is severely disturbed in trichothiodystrophy in contrast to general transcription deficiencies (whole genome).

A study comparing XP versus TTD mutations in the helicase XPD showed the following: all $\mathrm{XPD}$ mutations, regardless of causing Xeroderma pigmentosum or trichothiodystrophy are detrimental for XPD helicase activity, thus explaining the NER defect. TFIIH from TTD patients, but not from XP patients, exhibits a significant in vitro basal transcription defect in addition to a reduced intracellular concentration. Moreover, when XPD mutations prevent interaction with the p44 subunit of TFIIH, transactivation directed by certain nuclear receptors is inhibited, regardless of TTD versus XP phenotype, thus explaining the overlapping symptoms (Dubaele et al, 2003). TTD can also be caused by mutations in the TTDA subunit of TFIIH. Although this subunit is dispensable for the transcriptional activity of TFIIH in RNA polymerase II transcription, it nonetheless stabilizes TFIIH allowing expression of late acting genes and thus performs a specific gene expression activity (Hashimoto and Egly, 2009 and references therein).

Asking if TTD might be a transcription syndrome, the authors of the next study used microarrays to detect transcriptional differences between TTD and XP cells from the XP-D complementation group. They compared gene expression profiles in cultured fibroblasts from normal, XP and TTD donors and concluded that there are minimal differences in gene expression in proliferating fibroblasts from TTD, XP-D and normal donors (Offmann et al, 2008) thus arguing against transcriptional deficiencies being causal for trichothiodystrophy.

Mutations in XPB and XPD subunits of TFIIH can also cause a combination of xeroderma pigmentosum and Cockayne syndrome. As the failure of the DNA repair function of TFIIH explains the cancer susceptibility of xeroderma pigmentosum, additional functions of TFIIH might be responsible for the premature aging phenotype of Cockayne syndrome.

An optimized cell-free in vitro RNA polymerase II transcription assay was used to analyze transcription activity of XP-B and XP-D as well as XPB/CS and XPD/CS. Although the growth rate was normal, the XP-B and XP-D cells contained reduced amounts of TFIIH. Extracts prepared from XP-B and XP-D lymphoblastoid cells exhibited similar transcription activity from the adenovirus major late promoter when compared to that in extracts from 
normal cells. Thus, the authors concluded that the XP-B and XP-D lymphoblastoid cells do not have impaired RNA transcription activity. They considered the possible consequences of the reduced cellular content of TFIIH for the clinical symptoms in XP-B or XP-D patients, and discuss a 'conditional phenotype' that may involve an impairment of cellular function only under certain growth conditions (Satoh and Hanawald, 1997).

Subsequent another study investigated mutant TFIIH in a reconstituted RNA polymerase II transcription assay. Mutations in XP-B/Cockayne syndrome patients decrease the transcriptional activity of the corresponding TFIIH by preventing promoter opening of RNA polymerase II. The XP-B patient with the most severe symptoms was the patient with the lowest TFIIH transcription activity in vitro. These finding points out that the severity of the clinical symptoms observed within the XP-B patients is a function of the TFIIH activity in transcription rather than in NER. Both XPB mutations result in an almost total inhibition ( $95 \%)$ of NER. Western blot analysis and enzymatic assays indicate that XPD mutations affect the stoichometric composition of TFIIH due to a weakness in the interaction between XPDCAK complex and the core TFIIH, resulting in a partial reduction of transcription activity. The authors concluded that XP-B and XP-D patients are more likely to suffer from transcription repair syndromes rather than DNA repair disorders (Coin et al, 1999). This report identified failures of mutant TFIIH acting on the adenovirus-major-late promoter representative for all RNA polymerase II genes. Thus it describes a general deficiency in gene expression rather than a gene specific effect expected to be causative for Cockayne syndrome. TFIIH does influence gene-specific transcription by the interaction with transcriptional regulators or by phosphorylation of transcription factors like nuclear receptors (reviewed in Zurita and Merino, 2003).

\subsubsection{XPG in transcription}

XPG also called ERCC5 is a endonuclease that excises the 3`end of an unwinded damaged DNA single strand in nucleotide excision repair (NER). Endonuclease inactivating mutations are followed by xeroderma pigmentosum, whereas truncating mutations in XPG are followed by a severe form of Cockayne syndrome. The authors hypothesised that XPG exhibits a second function critical for the development of Cockayne syndrome (Nouspikel et al, 1997). That XPG as well as its yeast counterpart RAD2 are biochemically isolatable in a complex with TFIIH and interact with multiple subunits of this DNA repair/basal transcription factor was early recognized (Iyer et al,1996, Habraken et al, 1996). Genetic studies in yeast cells, knocking out the yeast counterparts of XPG and CSB, Rad2 and Rad26, unravelled an involvement of both proteins in transcription by RNA polymerase II.

The authors provide evidence for the involvement of RAD2 in Pol II-dependent transcription. Interestingly, they found that both transcription and growth are more severely inhibited in the rad $2 \Delta$ rad $26 \Delta$ double mutant than in the rad $2 \Delta$ and rad26 single mutants. These results indicate that $R A D 2$ and $R A D 26$ provide alternate means for efficient transcription, and further, they implicate transcriptional defects as the underlying cause of growth impairment that occurs in the $\operatorname{rad} 2 \Delta, \operatorname{rad} 26 \Delta$, and $\mathrm{rad} 2 \Delta \mathrm{rad} 26 \Delta$ mutant strains under conditions that would require the synthesis of new mRNAs. From these studies, they infer that CS is likely a transcription syndrome and that growth and developmental defects in CS could result from defects in transcription (Lee et al, 2002). In a report studying the involvement of CSB in RNA polymerase I transcription, functional XPG was identified in a complex with CSB, TFIIH, RNA polymerase I initiation factor TIF-IB and RNA polymerase I indicating that XPG might play a role in ribosomal transcription by RNA polymerase I 
(Bradsher et al, 2002). Thorel and coworkers described a mild case of Cockayne syndrome characterised by a XPG with nuclease activity that lost the interaction domain with TFIIH. This interaction might be critical for the development of the disease (Thorel et al, 2004).

Another level of complexity was added by the description of an epigenetic function of XPG. DNA methylation is an epigenetic modification that is essential for gene silencing and genome stability in many organisms. The authors show that Gadd45a (growth arrest and DNA-damage-inducible protein 45 alpha), a nuclear protein involved in maintenance of genomic stability, DNA repair and suppression of cell growth, has a key role in active DNA demethylation. Active demethylation occurs by DNA repair and Gadd45a interacts with and requires the DNA repair endonuclease XPG. They concluded that Gadd45a relieves epigenetic gene silencing by promoting DNA repair, which erases methylation marks (Barreto et al, 2007).

XPG forms a stable complex with TFIIH, which is active in transcription and NER. Mutations in XPG found in XP-G/CS patient cells that prevent the association with TFIIH also resulted in the dissociation of CAK and XPD from the core TFIIH. As a consequence, the phosphorylation and transactivation of nuclear receptors were disturbed in XP-G/CS as well as $x p g(-/-)$ MEF cells and could be restored by expression of wild-type XPG. These results provide an insight into the role of XPG in the stabilization of TFIIH and the regulation of gene expression and provide an explanation of some of the clinical features of XP-G/CS. (Ito et al, 2007). This is the first report indicating that XPG serves a gene-specific regulatory function in transcription. An involvement of XPG in the regulation of RNA polymerase I transcription described a mechanism that seems to be conserved between RNA polymerase I and II. In both cases, Gadd45a recruits the NER proteins including XPG to demethylate and activate epigenetic silenced promoter regions The results reveal a mechanism that recruits the DNA repair machinery to the promoter of active genes, keeping them in a hypomethylated state (Barreto et al, 2007; Schmitz et al, 2009). An intimate functional link between Nucleotide excision repair (NER) and transcription by RNA polymerase II was unravelled in the groundbreaking study by Egly and co-workers.

Upon gene activation, they found that RNA polymerase II transcription machinery assembles sequentially with the nucleotide excision repair (NER) factors at the promoter. This recruitment occurs in absence of exogenous genotoxic attack, is sensitive to transcription inhibitors, and depends on the XPC protein. The presence of these repair proteins at the promoter of activated genes is necessary in order to achieve optimal DNA demethylation and histone posttranslational modifications (H3K4/H3K9 methylation, H3K9/14 acetylation) and thus efficient RNA synthesis. Deficiencies in some NER factors impede the recruitment of others and affect nuclear receptor transactivation. This data suggest that there is a functional difference between the presence of the NER factors at the promoters (which requires XPC) and the NER factors at the distal regions of the gene (which requires CSB). While the latter may be a repair function, the former is a function with respect to transcription (LeMay et al, 2010).

\subsection{Hutchinson-Gilford progeria syndrome}

Hutchinson-Gilford progeria syndrome (HGPS) is a very rare genetic disorder with an estimated incidence rate of 1 in 8 million. Taken in consideration misdiagnosed or unreported cases, the true figure might be closer to 1 in 4 million (Pollex et al, 2004). HGPS was first described by Dr. Jonathan Hutchinson in 1886 and Dr. Hastings Gilford in 1897 and ever since just over 100 cases of HGPS have been reported. Like all progeria HGPS is 
characterised by segmental premature aging. Children with this disease appear normal at birth but manifestations of HGPS appear at the age between 12-24 months. Characteristic features include delayed dentition, micrognathia, loss of subcutaneous fat, growth retardation, midface hypoplasia, alopecia, atherosclerosis and generalised osteodysplasia with osteolysis and pathologic fractures (www.progeriaresearch.org). The median age at death is about 13 years, and at least $90 \%$ of all patients die from progressive atherosclerosis of the coronary and cerebrovascular arteries (Baker et al. 1981).

HGPS is caused by mutations in LMNA which encodes lamins A and C. Most patients $(80 \%)$ reveal a de novo heterozygous point mutation (G608G: GGC $\rightarrow$ GGT) in exon 11 of LMNA gene. (Eriksson et al, 2003; De Sandre-Giovannoli et al, 2003). Lamins A and C are type V intermediate filaments which are major components of the nuclear lamina, a protein scaffold at the inner nuclear membrane, which also extends as a network throughout the nucleus.

However, the LMNA G608G mutation responsible for most cases of HGPS does not cause an amino acid change, but activates a cryptic splice site leading to a truncated variant of lamin A (progerin) with an in-frame deletion of 50 amino acids near the carboxy terminus (Eriksson et al, 2003; De Sandre-Giovannoli et al, 2003). Due to the loss of 50 amino acids, progerin is lacking an important endoprotease cleavage site required for excision of the farnesylcystein methyl ester. Thus, the HGPS mutation causes the accumulation of permanently farnesylated progerin in the cell nucleus.

One of the most apparent outcomes of the accumulation of progerin is the morphological change of nuclei. HGPS is characterised by significant changes in nuclear size and shape, including lobulation of the nuclear envelope, wrinkle formation, thickening of the the nuclear lamina and clustering of nuclear pores (Goldman et al, 2004.; Eriksson et al, 2003; De Sandre-Giovannoli et al, 2003; Scaffidi et al, 2005;Lammerding et al, 2005).

Another aspect widely discussed in literature is the potential regulatory role of progerin in gene expression as lamins are also interacting with chromatin. Chromatin in Zmpste24MEFs, which are also accumulating farnesylated prelamin A (progerin), aggregates at discrete regions in a balloon shape and further analysis showed a variety of chromosomal abnormalities (aneuploidy, ring structures, chromosome instability and DNA breaks) (Liu et al, 2005). Further studies revealed that HGPS cells show significant changes in epinetic control of heterochromatin (Goldman et al, 2004; Shumaker et al, 2006). Heterochromatin markers such as histone $\mathrm{H} 3$ trimethylated on lysine 27 (H3K27me3), for facultative heterochromatin, as well as $\mathrm{H} 3$ trimethylated on lysine 9 (H3K9me3), for pericentric constitutive heterochromatin, are lost in HGPS cells (Shumakers et al, 2006). These changes could be directly link to progerin expression and are detectable even before changes in shapes of nuclei occur, suggesting that progerin changes gene regulation and silencing even at low levels.

It is suggested that lamin A has diverse roles in DNA metabolism, including DNA replication and transcription and also gene expression. Genome expression profiling of HGPS revealed differentially expressed genes in HGPS fibroblasts compared to age matched control cell lines, which play a role in a variety of biological processes. The most prominent categories encode transcription factors and extracellular matrix proteins, many of which are known to function in the tissues severely affected in HGPS. The most affected gene was MEOX/GAX, a homeobox gene that functions as a negative regulator of mesodermal tissue proliferation (Csoka et al, 2004). Microarray analysis from another study showed significant changes in 352 genes of which 306 were down regulated and 46 up regulated in HGPS cells. Functional analysis indicated that most of the genes are important for lipid metabolism, cell 
growth and differentiation, cell cycle, DNA replication and repair as well as cardiovascular system development (Marji et al, 2010). The only altered expressed gene encoding a protein known to directly interact with A-type lamins has been $R b 1$. Rb plays an important role in cell cycle control and also regulates differentiation. The level of $\mathrm{Rb}$ expression at the mRNA and protein level was down regulated in cells derived from HGPS patients and also downstream targets of $R b 1$ were affected (Marji et al, 2010). There is also evidence for a significant reduction of hyperphosphorylated Rb in HGPS fibroblasts (Dechat et al, 2007). Based on these observations, decreased $\mathrm{Rb}$ expression and reduction of hyperphosphorylated $\mathrm{Rb}$ in HGPS cells may lead to deregulation of proliferation (Marji et al, 2010). Similar observation have been reported in cells derived from Lmna $-/-$ mice implicating that absence or mutation of lamin $\mathrm{A}$ lead to unstable $\mathrm{Rb}$ and a altered lamin A/C-Rb signaling in HGPS cells (Johnson et al, 2004).

Transcription is also in part regulated by the nuclear scaffold which regulates the association and organisation of genes and transcription factors. Certain observations implicate that active transcription complexes are bound to the nuclear lamina and transcription factors as well as active genes are reported to be enriched in nuclear matrix preparations (Jackson et al, 1985; Stein et al, 1995). The contribution of nuclear lamins in transcription has been suggested by different studies. Loss of function mutation of lamin $\left(\mathrm{Dm}_{0}\right)$ in Drosophila disrupts the directed outgrowth of cytoplasmic extensions from terminal cells of the tracheal system, and oocytes from germ line mutants show improper localization of mRNA in the cytoplasm (Guillemin et al., 2001). These results confirm the requirement of nuclear lamin for cytoplasmic as well as nuclear organization. Lamin associated protein $2 \mathrm{~B}$ (LAP 2B) is a lamin binding protein and has been shown to mediate transcriptional repression (Mancini et al, 1994). Another study revealed that during vertebrate development, changes in the expression of lamins correlates with the beginning of transcription and cell differentiation (Moir et al, 1995). Additionally, disruption of normal lamin organization in active embryonic nuclei from Xenopus leads to inhibition of RNA polymerase II activity. The authors suggested that lamins may act as a scaffold upon which the basal transcription factors required for RNA polymerase II transcription are organized (Spann et al, 2002). A recent study by Osorio et al. also demonstrate reduction of RNA polymerase I transcription in Zmpst24 deficient mice. This decrease is due to hypermethylation and hypoacetylation of rDNA leading to a more compact, silent and dysfunctional rDNA gene activity (Osorio et al, 2010). It is also reported that DNA replication can be regulated by the lamina scaffold. Nuclei from Xenopus eggs lost their ability to synthesise DNA after immunodepletion of lamins (Newport et al, 1990). Furthermore, mutation in lamina in Xenopus blocks DNA replication at the transition from the initiation to the elongation phase of DNA replication (Moir et al, 2000).

\section{Conclusion}

Transcriptional alterations as the driving force behind premature aging- the implications are vast. The dispute of the competing hypothesis if its primarily unrepaired, accumulating DNA damage or if there is a kind of genetic program that drives premature and normal aging is not solved yet. As both pathways, the DNA damage response and transcriptional regulation are intimately connected by bi- or multifunctional proteins, the separation of progerias in DNA-repair or transcriptional syndromes might turn out to be artificial. The gene expression profiles, the transcriptomes of the aging body might be initiated by DNA 
damage but executed by specific transcription factors that respond to DNA damage. Mutations in these specific transcription factors could initiate a gene expression profile that resembles the normal answer to accumulating DNA damage. To prove these hypothesis there is a lot of exciting work ahead.

\section{Acknowlegdement}

We thank Sylvia Koch and Omar Garcia Gonzalez for stimulating discussions and Meinhard Wlaschek and Karin Scharffetter-Kochanek for continuos support. R.A. and S.I. are supported by a Grant of the German Research Society (DFG), Klinische Forschergruppe KFO142.

\section{References}

Baird, D.M., Davis, T., Rowson, J., Jones, C.J. and Kipling, D. (2004) Normal telomere erosion rates at the single cell level in Werner syndrome fibroblast cells. Hum Mol Genet, 13, 1515-1524.

Baker, P.B., Baba, N. and Boesel, C.P. (1981) Cardiovascular abnormalities in progeria. Case report and review of the literature. Arch Pathol Lab Med, 105, 384-386.

Barreto, G., Schafer, A., Marhold, J., Stach, D., Swaminathan, S.K., Handa, V., Doderlein, G., Maltry, N., Wu, W., Lyko, F. and Niehrs, C. (2007) Gadd45a promotes epigenetic gene activation by repair-mediated DNA demethylation. Nature, 445, 671-675.

Bergmann, E. and Egly, J.M. (2001) Trichothiodystrophy, a transcription syndrome. Trends Genet, 17, 279-286.

Blander, G., Kipnis, J., Leal, J.F., Yu, C.E., Schellenberg, G.D. and Oren, M. (1999) Physical and functional interaction between p53 and the Werner's syndrome protein. J Biol Chem, 274, 29463-29469.

Chalut, C., Moncollin, V. and Egly, J.M. (1994) Transcription by RNA polymerase II: a process linked to DNA repair. Bioessays, 16, 651-655.

Chang, S., Multani, A.S., Cabrera, N.G., Naylor, M.L., Laud, P., Lombard, D., Pathak, S., Guarente, L. and DePinho, R.A. (2004) Essential role of limiting telomeres in the pathogenesis of Werner syndrome. Nat Genet, 36, 877-882.

Charlet-Berguerand, N., Feuerhahn, S., Kong, S.E., Ziserman, H., Conaway, J.W., Conaway, R. and Egly, J.M. (2006) RNA polymerase II bypass of oxidative DNA damage is regulated by transcription elongation factors. Embo J, 25, 5481-5491.

Chu, W.K. and Hickson, I.D. (2009) RecQ helicases: multifunctional genome caretakers. Nat Rev Cancer, 9, 644-654.

Coin, F., Bergmann, E., Tremeau-Bravard, A. and Egly, J.M. (1999) Mutations in XPB and $\mathrm{XPD}$ helicases found in xeroderma pigmentosum patients impair the transcription function of TFIIH. Embo J, 18, 1357-1366.

Crabbe, L., Jauch, A., Naeger, C.M., Holtgreve-Grez, H. and Karlseder, J. (2007) Telomere dysfunction as a cause of genomic instability in Werner syndrome. Proc Natl Acad Sci U S A, 104, 2205-2210.

Crabbe, L., Verdun, R.E., Haggblom, C.I. and Karlseder, J. (2004) Defective telomere lagging strand synthesis in cells lacking WRN helicase activity. Science, 306, 1951-1953.

Csoka, A.B., English, S.B., Simkevich, C.P., Ginzinger, D.G., Butte, A.J., Schatten, G.P., Rothman, F.G. and Sedivy, J.M. (2004) Genome-scale expression profiling of 
Hutchinson-Gilford progeria syndrome reveals widespread transcriptional misregulation leading to mesodermal/mesenchymal defects and accelerated atherosclerosis. Aging Cell, 3, 235-243.

de Boer, J., Andressoo, J.O., de Wit, J., Huijmans, J., Beems, R.B., van Steeg, H., Weeda, G., van der Horst, G.T., van Leeuwen, W., Themmen, A.P., Meradji, M. and Hoeijmakers, J.H. (2002) Premature aging in mice deficient in DNA repair and transcription. Science, 296, 1276-1279.

De Sandre-Giovannoli, A., Bernard, R., Cau, P., Navarro, C., Amiel, J., Boccaccio, I., Lyonnet, S., Stewart, C.L., Munnich, A., Le Merrer, M. and Levy, N. (2003) Lamin a truncation in Hutchinson-Gilford progeria. Science, 300, 2055.

Dechat, T., Shimi, T., Adam, S.A., Rusinol, A.E., Andres, D.A., Spielmann, H.P., Sinensky, M.S. and Goldman, R.D. (2007) Alterations in mitosis and cell cycle progression caused by a mutant lamin A known to accelerate human aging. Proc Natl Acad Sci U $S$ A, 104, 4955-4960.

Dianov, G.L., Houle, J.F., Iyer, N., Bohr, V.A. and Friedberg, E.C. (1997) Reduced RNA polymerase II transcription in extracts of cockayne syndrome and xeroderma pigmentosum/Cockayne syndrome cells. Nucleic Acids Res, 25, 3636-3642.

Douziech, M., Coin, F., Chipoulet, J.M., Arai, Y., Ohkuma, Y., Egly, J.M. and Coulombe, B. (2000) Mechanism of promoter melting by the xeroderma pigmentosum complementation group B helicase of transcription factor IIH revealed by proteinDNA photo-cross-linking. Mol Cell Biol, 20, 8168-8177.

Drapkin, R., Reardon, J.T., Ansari, A., Huang, J.C., Zawel, L., Ahn, K., Sancar, A. and Reinberg, D. (1994) Dual role of TFIIH in DNA excision repair and in transcription by RNA polymerase II. Nature, 368, 769-772.

Eriksson, M., Brown, W.T., Gordon, L.B., Glynn, M.W., Singer, J., Scott, L., Erdos, M.R., Robbins, C.M., Moses, T.Y., Berglund, P., Dutra, A., Pak, E., Durkin, S., Csoka, A.B., Boehnke, M., Glover, T.W. and Collins, F.S. (2003) Recurrent de novo point mutations in lamin A cause Hutchinson-Gilford progeria syndrome. Nature, 423, 293-298.

Faragher, R.G., Kill, I.R., Hunter, J.A., Pope, F.M., Tannock, C. and Shall, S. (1993) The gene responsible for Werner syndrome may be a cell division "counting" gene. Proc Natl Acad Sci U S A, 90, 12030-12034.

Filippi, S., Latini, P., Frontini, M., Palitti, F., Egly, J.M. and Proietti-De-Santis, L. (2008) CSB protein is (a direct target of HIF-1 and) a critical mediator of the hypoxic response. Embo J, 27, 2545-2556.

Goldman, R.D., Shumaker, D.K., Erdos, M.R., Eriksson, M., Goldman, A.E., Gordon, L.B., Gruenbaum, Y., Khuon, S., Mendez, M., Varga, R. and Collins, F.S. (2004) Accumulation of mutant lamin A causes progressive changes in nuclear architecture in Hutchinson-Gilford progeria syndrome. Proc Natl Acad Sci U S A, 101, 8963-8968.

Guillemin, K., Williams, T. and Krasnow, M.A. (2001) A nuclear lamin is required for cytoplasmic organization and egg polarity in Drosophila. Nat Cell Biol, 3, 848-851.

Guzder, S.N., Sung, P., Bailly, V., Prakash, L. and Prakash, S. (1994) RAD25 is a DNA helicase required for DNA repair and RNA polymerase II transcription. Nature, $369,578-581$. 
Habraken, Y., Sung, P., Prakash, S. and Prakash, L. (1996) Transcription factor TFIIH and DNA endonuclease Rad2 constitute yeast nucleotide excision repair factor 3: implications for nucleotide excision repair and Cockayne syndrome. Proc Natl Acad Sci U S A, 93, 10718-10722.

Hashimoto, S. and Egly, J.M. (2009) Trichothiodystrophy view from the molecular basis of DNA repair/transcription factor TFIIH. Hum Mol Genet, 18, R224-230.

Henning, K.A., Li, L., Iyer, N., McDaniel, L.D., Reagan, M.S., Legerski, R., Schultz, R.A., Stefanini, M., Lehmann, A.R., Mayne, L.V. and Friedberg, E.C. (1995) The Cockayne syndrome group A gene encodes a WD repeat protein that interacts with CSB protein and a subunit of RNA polymerase II TFIIH. Cell, 82, 555-564.

Herbig, U., Ferreira, M., Condel, L., Carey, D. and Sedivy, J.M. (2006) Cellular senescence in aging primates. Science, 311, 1257.

Hoeijmakers, J.H. (2009) DNA damage, aging, and cancer. N Engl J Med, 361, 1475-1485.

Horibata, K., Iwamoto, Y., Kuraoka, I., Jaspers, N.G., Kurimasa, A., Oshimura, M., Ichihashi, M. and Tanaka, K. (2004) Complete absence of Cockayne syndrome group B gene product gives rise to UV-sensitive syndrome but not Cockayne syndrome. Proc Natl Acad Sci U S A, 101, 15410-15415.

Iben, S., Tschochner, H., Bier, M., Hoogstraten, D., Hozak, P., Egly, J.M. and Grummt, I. (2002) TFIIH plays an essential role in RNA polymerase I transcription. Cell, 109, 297-306.

Ito, S., Kuraoka, I., Chymkowitch, P., Compe, E., Takedachi, A., Ishigami, C., Coin, F., Egly, J.M. and Tanaka, K. (2007) XPG stabilizes TFIIH, allowing transactivation of nuclear receptors: implications for Cockayne syndrome in XP-G/CS patients. Mol Cell, 26, 231-243.

Iyer, N., Reagan, M.S., Wu, K.J., Canagarajah, B. and Friedberg, E.C. (1996) Interactions involving the human RNA polymerase II transcription/nucleotide excision repair complex TFIIH, the nucleotide excision repair protein XPG, and Cockayne syndrome group B (CSB) protein. Biochemistry, 35, 2157-2167.

Jackson, D.A. and Cook, P.R. (1985) Transcription occurs at a nucleoskeleton. Embo J, 4, 919925.

Johnson, B.R., Nitta, R.T., Frock, R.L., Mounkes, L., Barbie, D.A., Stewart, C.L., Harlow, E. and Kennedy, B.K. (2004) A-type lamins regulate retinoblastoma protein function by promoting subnuclear localization and preventing proteasomal degradation. Proc Natl Acad Sci U S A, 101, 9677-9682.

Johnson, J.E., Cao, K., Ryvkin, P., Wang, L.S. and Johnson, F.B. Altered gene expression in the Werner and Bloom syndromes is associated with sequences having Gquadruplex forming potential. Nucleic Acids Res, 38, 1114-1122.

Kim, T.K., Ebright, R.H. and Reinberg, D. (2000) Mechanism of ATP-dependent promoter melting by transcription factor IIH. Science, 288, 1418-1422.

Kirkwood, T.B. Global aging and the brain. Nutr Rev, 68 Suppl 2, S65-69.

Kyng, K.J., May, A., Brosh, R.M., Jr., Cheng, W.H., Chen, C., Becker, K.G. and Bohr, V.A. (2003) The transcriptional response after oxidative stress is defective in Cockayne syndrome group B cells. Oncogene, 22, 1135-1149.

Lammerding, J., Hsiao, J., Schulze, P.C., Kozlov, S., Stewart, C.L. and Lee, R.T. (2005) Abnormal nuclear shape and impaired mechanotransduction in emerin-deficient cells. J Cell Biol, 170, 781-791. 
Laugel, V., et al., (2009) Mutation update for the CSB/ERCC6 and CSA/ERCC8 genes involved in Cockayne syndrome. Hum Mutat, 31, 113-126.

Le May, N., Mota-Fernandes, D., Velez-Cruz, R., Iltis, I., Biard, D. and Egly, J.M. NER factors are recruited to active promoters and facilitate chromatin modification for transcription in the absence of exogenous genotoxic attack. Mol Cell, 38, 54-66.

Le May, N., Mota-Fernandes, D., Velez-Cruz, R., Iltis, I., Biard, D. and Egly, J.M. NER factors are recruited to active promoters and facilitate chromatin modification for transcription in the absence of exogenous genotoxic attack. Mol Cell, 38, 54-66.

Lebedev, A., Scharffetter-Kochanek, K. and Iben, S. (2008) Truncated Cockayne syndrome B protein represses elongation by RNA polymerase I. J Mol Biol, 382, 266-274.

Lee, S.K., Johnson, R.E., Yu, S.L., Prakash, L. and Prakash, S. (1999) Requirement of yeast SGS1 and SRS2 genes for replication and transcription. Science, 286, 2339-2342.

Lee, S.K., Yu, S.L., Prakash, L. and Prakash, S. (2001) Requirement for yeast RAD26, a homolog of the human CSB gene, in elongation by RNA polymerase II. Mol Cell Biol, 21, 8651-8656.

Lee, S.K., Yu, S.L., Prakash, L. and Prakash, S. (2002) Requirement of yeast RAD2, a homolog of human XPG gene, for efficient RNA polymerase II transcription. implications for Cockayne syndrome. Cell, 109, 823-834.

Lee, S.K., Yu, S.L., Prakash, L. and Prakash, S. (2002) Yeast RAD26, a homolog of the human CSB gene, functions independently of nucleotide excision repair and base excision repair in promoting transcription through damaged bases. Mol Cell Biol, 22, 43834389.

Liu, B., Wang, J., Chan, K.M., Tjia, W.M., Deng, W., Guan, X., Huang, J.D., Li, K.M., Chau, P.Y., Chen, D.J., Pei, D., Pendas, A.M., Cadinanos, J., Lopez-Otin, C., Tse, H.F., Hutchison, C., Chen, J., Cao, Y., Cheah, K.S., Tryggvason, K. and Zhou, Z. (2005) Genomic instability in laminopathy-based premature aging. Nat Med, 11, 780-785.

Lutomska, A., Lebedev, A., Scharffetter-Kochanek, K. and Iben, S. (2008) The transcriptional response to distinct growth factors is impaired in Werner syndrome cells. Exp Gerontol, 43, 820-826.

Mancini, M.A., Shan, B., Nickerson, J.A., Penman, S. and Lee, W.H. (1994) The retinoblastoma gene product is a cell cycle-dependent, nuclear matrix-associated protein. Proc Natl Acad Sci U S A, 91, 418-422.

Marji, J., O'Donoghue, S.I., McClintock, D., Satagopam, V.P., Schneider, R., Ratner, D., Worman, H.J., Gordon, L.B. and Djabali, K. Defective lamin A-Rb signaling in Hutchinson-Gilford Progeria Syndrome and reversal by farnesyltransferase inhibition. PLoS One, 5, e11132.

Moir, R.D., Spann, T.P. and Goldman, R.D. (1995) The dynamic properties and possible functions of nuclear lamins. Int Rev Cytol, 162B, 141-182.

Moir, R.D., Spann, T.P., Herrmann, H. and Goldman, R.D. (2000) Disruption of nuclear lamin organization blocks the elongation phase of DNA replication. J Cell Biol, 149, 1179-1192.

Nance, M.A. and Berry, S.A. (1992) Cockayne syndrome: review of 140 cases. Am J Med Genet, 42, 68-84.

Newman, J.C., Bailey, A.D. and Weiner, A.M. (2006) Cockayne syndrome group B protein (CSB) plays a general role in chromatin maintenance and remodeling. Proc Natl Acad Sci U S A, 103, 9613-9618. 
Newport, J.W., Wilson, K.L. and Dunphy, W.G. (1990) A lamin-independent pathway for nuclear envelope assembly. J Cell Biol, 111, 2247-2259.

Nouspikel, T., Lalle, P., Leadon, S.A., Cooper, P.K. and Clarkson, S.G. (1997) A common mutational pattern in Cockayne syndrome patients from xeroderma pigmentosum group G: implications for a second XPG function. Proc Natl Acad Sci U S A, 94, 31163121.

Osorio, F.G., Varela, I., Lara, E., Puente, X.S., Espada, J., Santoro, R., Freije, J.M., Fraga, M.F. and Lopez-Otin, $\mathrm{C}$. Nuclear envelope alterations generate an aging-like epigenetic pattern in mice deficient in Zmpste24 metalloprotease. Aging Cell, 9, 947-957.

Pollex, R.L. and Hegele, R.A. (2004) Hutchinson-Gilford progeria syndrome. Clin Genet, 66, 375-381.

Proietti-De-Santis, L., Drane, P. and Egly, J.M. (2006) Cockayne syndrome B protein regulates the transcriptional program after UV irradiation. Embo J, 25, 1915-1923.

Rossi, M.L., Ghosh, A.K. and Bohr, V.A. Roles of Werner syndrome protein in protection of genome integrity. DNA Repair (Amst), 9, 331-344.

Satoh, M.S. and Hanawalt, P.C. (1997) Competent transcription initiation by RNA polymerase II in cell-free extracts from xeroderma pigmentosum groups B and D in an optimized RNA transcription assay. Biochim Biophys Acta, 1354, 241-251.

Scaffidi, P., Gordon, L. and Misteli, T. (2005) The cell nucleus and aging: tantalizing clues and hopeful promises. PLoS Biol, 3, e395.

Schmitz, K.M., Schmitt, N., Hoffmann-Rohrer, U., Schafer, A., Grummt, I. and Mayer, C. (2009) TAF12 recruits Gadd45a and the nucleotide excision repair complex to the promoter of rRNA genes leading to active DNA demethylation. Mol Cell, 33, 344353.

Schmitz, K.M., Schmitt, N., Hoffmann-Rohrer, U., Schafer, A., Grummt, I. and Mayer, C. (2009) TAF12 recruits Gadd45a and the nucleotide excision repair complex to the promoter of rRNA genes leading to active DNA demethylation. Mol Cell, 33, 344353.

Selby, C.P. and Sancar, A. (1997) Cockayne syndrome group B protein enhances elongation by RNA polymerase II. Proc Natl Acad Sci U S A, 94, 11205-11209.

Shiratori, M., Suzuki, T., Itoh, C., Goto, M., Furuichi, Y. and Matsumoto, T. (2002) WRN helicase accelerates the transcription of ribosomal RNA as a component of an RNA polymerase I-associated complex. Oncogene, 21, 2447-2454.

Shumaker, D.K., Dechat, T., Kohlmaier, A., Adam, S.A., Bozovsky, M.R., Erdos, M.R., Eriksson, M., Goldman, A.E., Khuon, S., Collins, F.S., Jenuwein, T. and Goldman, R.D. (2006) Mutant nuclear lamin A leads to progressive alterations of epigenetic control in premature aging. Proc Natl Acad Sci U S A, 103, 8703-8708.

Sinclair, D.A., Mills, K. and Guarente, L. (1997) Accelerated aging and nucleolar fragmentation in yeast sgs1 mutants. Science, 277, 1313-1316.

Spann, T.P., Goldman, A.E., Wang, C., Huang, S. and Goldman, R.D. (2002) Alteration of nuclear lamin organization inhibits RNA polymerase II-dependent transcription. J Cell Biol, 156, 603-608.

Stein, G.S., van Wijnen, A.J., Stein, J., Lian, J.B. and Montecino, M. (1995) Contributions of nuclear architecture to transcriptional control. Int Rev Cytol, 162A, 251-278. 
Tantin, D., Kansal, A. and Carey, M. (1997) Recruitment of the putative transcription-repair coupling factor CSB/ERCC6 to RNA polymerase II elongation complexes. Mol Cell Biol, 17, 6803-6814.

Thorel, F., Constantinou, A., Dunand-Sauthier, I., Nouspikel, T., Lalle, P., Raams, A., Jaspers, N.G., Vermeulen, W., Shivji, M.K., Wood, R.D. and Clarkson, S.G. (2004) Definition of a short region of XPG necessary for TFIIH interaction and stable recruitment to sites of UV damage. Mol Cell Biol, 24, 10670-10680.

Troelstra, C., van Gool, A., de Wit, J., Vermeulen, W., Bootsma, D. and Hoeijmakers, J.H. (1992) ERCC6, a member of a subfamily of putative helicases, is involved in Cockayne's syndrome and preferential repair of active genes. Cell, 71, 939-953.

Turaga, R.V., Paquet, E.R., Sild, M., Vignard, J., Garand, C., Johnson, F.B., Masson, J.Y. and Lebel, M. (2009) The Werner syndrome protein affects the expression of genes involved in adipogenesis and inflammation in addition to cell cycle and DNA damage responses. Cell Cycle, 8, 2080-2092.

van Gool, A.J., Citterio, E., Rademakers, S., van Os, R., Vermeulen, W., Constantinou, A., Egly, J.M., Bootsma, D. and Hoeijmakers, J.H. (1997) The Cockayne syndrome B protein, involved in transcription-coupled DNA repair, resides in an RNA polymerase II-containing complex. Embo J, 16, 5955-5965.

Winkler, G.S. and Hoeijmakers, J.H. (1998) From a DNA helicase to brittle hair. Nat Genet, 20, 106-107.

Yu, A., Fan, H.Y., Liao, D., Bailey, A.D. and Weiner, A.M. (2000) Activation of p53 or loss of the Cockayne syndrome group $\mathrm{B}$ repair protein causes metaphase fragility of human U1, U2, and 5S genes. Mol Cell, 5, 801-810.

Yuan, X., Feng, W., Imhof, A., Grummt, I. and Zhou, Y. (2007) Activation of RNA polymerase I transcription by cockayne syndrome group B protein and histone methyltransferase G9a. Mol Cell, 27, 585-595.

Zurita, M. and Merino, C. (2003) The transcriptional complexity of the TFIIH complex. Trends Genet, 19, 578-584. 


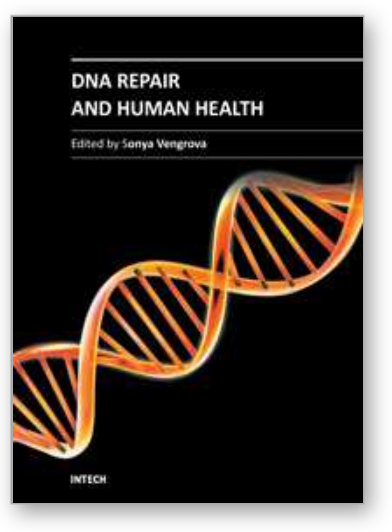

\author{
DNA Repair and Human Health \\ Edited by Dr. Sonya Vengrova
}

ISBN 978-953-307-612-6

Hard cover, 792 pages

Publisher InTech

Published online 26, October, 2011

Published in print edition October, 2011

Over the past decades, great advances have been made in understanding the cellular DNA repair pathways. At the same time, a wealth of descriptive knowledge of human diseases has been accumulated. Now, the basic research of the mechanisms of DNA repair is merging with clinical research, placing the action of the DNA repair pathways in the context of the whole organism. Such integrative approach enables understanding of the disease mechanisms and is invaluable in improving diagnostics and prevention, as well as designing better therapies. This book highlights the central role of DNA repair in human health and well-being. The reviews presented here, contain detailed descriptions of DNA repair pathways, as well as analysis of a large body of evidence addressing links between DNA damage repair and human health. They will be of interest to a broad audience, from molecular biologists working on DNA repair in any model system, to medical researchers.

\title{
How to reference
}

In order to correctly reference this scholarly work, feel free to copy and paste the following:

Robin Assfalg and Sebastian Iben (2011). Transcriptional Functions of DNA Repair Proteins Involved in Premature Aging, DNA Repair and Human Health, Dr. Sonya Vengrova (Ed.), ISBN: 978-953-307-612-6, InTech, Available from: http://www.intechopen.com/books/dna-repair-and-human-health/transcriptionalfunctions-of-dna-repair-proteins-involved-in-premature-aging

\section{INTECH}

open science | open minds

\section{InTech Europe}

University Campus STeP Ri

Slavka Krautzeka 83/A

51000 Rijeka, Croatia

Phone: +385 (51) 770447

Fax: +385 (51) 686166

www.intechopen.com

\section{InTech China}

Unit 405, Office Block, Hotel Equatorial Shanghai

No.65, Yan An Road (West), Shanghai, 200040, China

中国上海市延安西路65号上海国际贵都大饭店办公楼 405 单元

Phone: +86-21-62489820

Fax: $+86-21-62489821$ 
(C) 2011 The Author(s). Licensee IntechOpen. This is an open access article distributed under the terms of the Creative Commons Attribution 3.0 License, which permits unrestricted use, distribution, and reproduction in any medium, provided the original work is properly cited. 